\title{
Lungenkrebs in Zentraleuropa
}

Die Lungenkrebs-Prävalenz ist in Zentraleuropa im internationalen Vergleich sehr hoch ist. Der Austausch zwischen Lungenkrebs-Spezialisten in dieser Region ist daher von besonderer Relevanz, um der rasanten Entwicklung in der komplexen Diagnostik und Therapie dieser Erkrankung folgen zu können. Die 14th Central European Lung Cancer Conference, CELCC 2014 bot Ende November/Anfang Dezember in Wien den Rahmen dafür.

„Lungenkrebs gehört zu den dringlichsten Gesundheitsproblemen der Region“, betont Univ.-Prof. Dr. Robert Pirker, Universitätsklinik für Innere Medizin I, Medizinische Universität Wien. Lungenkrebs war 2012 mit rund 410.000 Neuerkrankungen die vierthäufigste Krebserkrankung, mit 353.000 Todesfällen aber die Krebsart, die die meisten Leben gekostet hat, zeigt eine im European Journal of Cancer veröffentlichte Arbeit über Krebserkrankungen in 40 europäischen Staaten. „Männer sind fast 2,5-mal so oft von Lungenkrebs betroffen wie Frauen und haben eine deutlich schlechtere Prognose“, so der CELCC 2014 Kongresspräsident Pirker. „Frauen holen allerdings in vielen Ländern auf alarmierende Weise auf, auch in Österreich. In einer steigenden Zahl europäischer Staaten sterben Frauen inzwischen häufiger an Lungen- als an Brustkrebs." Die Situation in den zentral- und osteuropäischen Staaten ist ähnlich bzw teilweise noch dramatischer.

„Etwa 85 Prozent der Betroffenen in Österreich und Zentraleuropa sind Raucher oder ehemalige Raucher", so OÄ Dr. Andrea Mohn-Staudner, SMZ Baumgartner Höhe, Wien. Lungenkrebs ließe sich also in einem in hohem Maße vermeiden. Auch Passivrauchen erhöht das Lungenkrebs-Risiko. „Dabei spielen nicht nur die kanzerogenen Substanzen im Nebenstromrauch eine Rolle, sondern auch die erhebliche Fein- staubbelastung in geschlossenen Räumen", so Mohn-Staudner.

\section{Screening von Risikogruppen senkt die Sterblichkeit}

Ein Problem bei der Behandlung von Lungenkrebs ist das späte Stadium, in dem die Erkrankung in der derzeit noch überwiegenden Zahl der Fälle diagnostiziert wird, da Symptome erst im weiteren Verlauf auftreten. „Da nur jene Patienten eine gute Prognose haben, bei denen der Tumor in einem sehr frühen Stadium diagnostiziert wird, ist die Früherkennung ein wichtiges Anliegen“, stellte Ass.-Prof. Dr. Helmut Prosch, Universitätsklinik für Radiologie und Nuklearmedizin der MedUniWien, fest. Daten aus dem in den USA durchgeführten National Lung Screening Trial (NLST) zufolge kann im Vergleich zu Röntgen-Vorsorgeuntersuchungen die Lungenkrebsmortalität durch ein Screening mittels NiedrigDosis-Spiral-CT (Low-Dose-CT, LD-CT) um 20 Prozent gesenkt werden. Wesentlich ist, nur Personen mit hohem Lungenkrebsrisiko zu erfassen, um eine hohe Zahl falsch-positiver Befunde oder eine unnötige Strahlenbelastung zu vermeiden.

\section{Molekulare Diagnostik und zielgerichtete Therapien}

Im Sinne einer präzisen Diagnostik können Instrumente auf molekularer Ebene Veränderungen in der Tumorzelle analysieren, um auf der Grundlage bestimmter Mutationen die zielgerichtete Therapie einzusetzen. So kommt dem epidermalen WachstumsfaktorRezeptor („,epidermal growth factor receptor", EGFR), bei Proliferation und Überleben der Tumorzelle eine entscheidende Bedeutung zu. EGFR-gerichtete Therapien agieren einerseits mit Tyrosinkinase-Inhibitoren (TKI), wobei sich drei Substanzen (Erlotinib, Gefitintib und Afatinib) bereits in thera- peutischen Settings etablierten konnten. „Untersucht wurden auch monoklonale Antikörper wie Cetuximab und Necitumumab", so Pirker. Bei metastasierenden Adenokarzinomen zählt die Bestimmung des EGFR- und des ALKMutationsstatus inzwischen zum klinischen Standard, wobei ALK für ein Gen der anaplastischen Lymphomkinase steht. „Die INSIGHT-Studie, die in sechs zentraleuropäischen Ländern durchgeführt wurde, bestätigt, dass die Bestimmung der EGFR-Mutationen zumindest in den größeren onkologischen Zentren zur klinischen Routine gehört und die Therapie in den untersuchten Staaten bereits nach den neuesten Qualitätskriterien erfolgt," erklärte Pirker:

Als weitere wichtige therapeutische Entwicklung gelten derzeit neue Möglichkeiten der Immuntherapie des Lungenkrebses, berichtete Pirker. „Insbesondere Ipilimumab, Anti-PD-1-und Anti-PD-L1-Antikörper geben in diesem Zusammenhang neue Hoffnung. Diese monoklonalen Antikörper werden derzeit in Phase-III-Studien beim Bronchialkarzinom untersucht. Fortschritte verzeichnen auch die multimodalen Therapien aus Chemo- und Strahlentherapie." Die adjuvante Chemotherapie, vorzugsweise in vier Zyklen mit Cisplatin und Vinorelbin angewandt, hat sich als Standardtherapie für Patienten herauskristallisiert, bei denen ein nichtkleinzelliges Lungenkarzinom vollständig entfernt worden ist. Sie kann die Überlebenschancen deutlich erhöhen. Eine weitere Verbesserung der Behandlungsergebnisse wird durch eine Integration zielgerichteter molekularer Therapien oder der Immuntherapie in die adjuvante Chemotherapie erwartet.

Wien klin Mag 2014 · 17:38 DOI 10.1007/s00740-014-0028-z Online publiziert: 9. Dezember 2014 (c) Springer-Verlag Wien 2014 